\title{
Distinguishing risk factors between congenital anophthalmia and microphthalmia using multivariable logistic regression
}

\author{
Yang Li, Zhijia Hou, Jingwen Ding, Ying Cui, Bixuan Qin, Dongmei Li \\ Beijing Tongren Eye Center, Beijing Tongren Hospital, Beijing Ophthalmology and Visual Science Key Lab, Capital Medical University, Beijing, \\ China \\ Contributions: (I) Conception and design: Y Li, D Li; (II) Administrative support: D Li; (III) Provision of study materials or patients: Z Hou, J Ding; (IV) \\ Collection and assembly of data: Y Cui, B Qin; (V) Data analysis and interpretation: Y Li; (VI) Manuscript writing: All authors; (VII) Final approval \\ of manuscript: All authors. \\ Correspondence to: Dongmei Li. Beijing Tongren Eye Center, Beijing Tongren Hospital, Beijing Ophthalmology and Visual Science Key Lab, Capital \\ Medical University, 1\# Dong Jiao Min Xiang, Beijing, China. Email: 1dmlily@x263.net.
}

\begin{abstract}
Background: Etiologies of congenital microphthalmia and anophthalmia are unclear and commonly thought to be homogenous. To test if risk factors are similar for these two diseases, we compared the risk factors between congenital microphthalmia and anophthalmia in a large Chinese cohort.

Methods: A total of 347 patients with congenital microphthalmia or anophthalmia diagnosed by magnetic resonance imaging (MRI), computed tomography (CT) or ultrasound from 2011 to 2018 were enrolled. Patients' clinical information, used as potential risk factors, was retrospectively collected. A multivariable logistic regression model was used to estimate odds ratios (OR) and 95\% confidence intervals (CI).

Results: A total of 347 patients were affected by congenital microphthalmia or anophthalmia. A total of 324 cases were microphthalmia, and 23 cases were anophthalmia. Structural abnormalities, mother's age at initial pregnancy, whether the mother drinks, whether the mother was diseased during pregnancy and whether the father has systemic disease passed the univariate test. In the multivariable logistic regression model, whether the mother was diseased during pregnancy ( $\mathrm{OR}=2.804, \mathrm{P}=0.023$ ) and whether the father had systemic disease $(\mathrm{OR}=4.795, \mathrm{P}=0.027)$ are significant risk factors for anophthalmia over microphthalmia. Influenza or common cold infection accounted most of the mother's diseases during pregnancy.

Conclusions: Mothers with diseases, mainly influenza or common cold infection, during pregnancy are more likely to have baby with anophthalmia than microphthalmia. Our study indicated that there might be different etiologies for anophthalmia and microphthalmia.
\end{abstract}

Keywords: Anophthalmia; microphthalmia; risk factor; multivariable logistic regression; influenza or common cold infection

Submitted Sep 16, 2019. Accepted for publication Dec 05, 2019.

doi: 10.21037/atm.2019.12.103

View this article at: http://dx.doi.org/10.21037/atm.2019.12.103

\section{Introduction}

Anophthalmia and microphthalmia are a set of rare, yet severe, birth defects that are thought to represent a phenotypic spectrum ranging from completely absent to smaller than average size eyes. Despite their clinical significance, their exact pathogenesis is not fully understood. It has been hypothesized that these malformations might occur when either the optic vesicles, anterior neural tube, or optic pits fail to develop early during embryonic development (approximately 6-10 weeks of gestation) or in the formation or closure of the fetal fissure (1). Moreover, abnormalities in the posterior segment of the eye, such as decreased optic cup size or low intraocular pressure, may contribute to the development of microphthalmia (1). Anophthalmia and microphthalmia can occur as part of 
recognized syndromes or in isolation (2).

Anophthalmia and microphthalmia are always evaluated together in epidemiologic studies because they might share common disrupted developmental pathways. However, it is possible that they may share distinct etiologies (3). The specific pathogeneses underlying anophthalmia and microphthalmia remain largely unknown. In previous studies, most population-based studies of these anomalies have used data gathered from routine reporting systems, such as congenital anomaly registers $(4,5)$. However, there are significant limitations to using these sources. When children are not examined by a specialist, diagnostic errors can be introduced. In our study, every patient was diagnosed by magnetic resonance imaging (MRI), computed tomography (CT) or ultrasound. We evaluated incomplete eyeball clearly from imaging to distinguish anophthalmia from microphthalmia. Furthermore, we first compared risk factors between patients with congenital microphthalmia and anophthalmia to discuss their possible distinct etiologies.

\section{Methods}

\section{Participants}

This clinical comparative study included 347 patients who were diagnosed with congenital anophthalmia and microphthalmia in Beijing Tongren Hospital from 2011 to 2018 in a consecutive manner. All patients were diagnosed by MRI, CT or ultrasound.

The study was approved by the Ethics Committee of Beijing Tongren Hospital and adhered to the provisions of the Declaration of Helsinki for research involving human subjects. Written informed consent was obtained from all adult participants. For the children, written informed consent was obtained from their parents.

\section{Case definition of microphthalmia and anophthalmia}

Anophthalmia was defined as no visible sign of a globe on CT scan or MRI. Microphthalmia was defined as "an abnormally small eye". Because not all children would undergo ultrasound to determine exact ocular dimensions, reference measurements for microphthalmia (axial length: $16 \mathrm{~mm}$ at birth, $19 \mathrm{~mm}$ at 12 months of age; corneal diameter: at birth $10 \mathrm{~mm}$ ) were provided as a guide only, not as diagnostic criteria.

The following diagnoses were explicitly excluded: eyelid coloboma, anterior segment anomalies (e.g., aniridia, Peters' anomaly), and other posterior noncolobomatous anomalies (e.g., retinopathy of prematurity, persistent fetal vasculature syndrome, and optic nerve hypoplasia).

\section{Study variables}

A standardized questionnaire was sent to all ophthalmologists who had notified an eligible child. The requested information included the date of birth, postal code, initials, sex twin/singleton (to exclude duplicate reporting), clinical phenotype, investigations undertaken, results, and clinical management.

Data were obtained from registration records on the following variables: infant sex, birth year, birth mode, delivery mode, race, place of birth, maternal age (pregnancy with patient), mother age (initial pregnancy), number of previous pregnancies, mother smoke, mother drink, mother with systemic disease, and mother with disease during pregnancy. The following paternal characteristics were obtained: age, smoke, drink and systemic disease.

\section{Statistical methods}

Descriptive statistics were calculated for continuous variables using the mean (SD) and for categorical data using $\mathrm{n}(\%)$. Data were analyzed using SPSS version 22.0 for Windows (IBM Corp., Armonk, NY, USA). We compared two groups using the $t$-test for continuous variables and the chi-square test for categorical variables. Pearson's chi-square test was used to test qualitative associations if all expected frequencies were equal to or greater than 5. Fisher's test was used when the expected frequencies were less than 5. A P value of 0.05 or less was used to define statistically significant results. All hypothesis tests used two-sided tests and set the alpha level at 0.05 . The variables considered important were included in a multivariable logistic regression model to estimate odds ratios (OR) and $95 \%$ confidence intervals (CI). Two-tailed $\mathrm{P}<0.05$ values were considered to indicate statistically significant differences.

\section{Results}

We accumulated a total of 347 cases of congenital microphthalmia and anophthalmia diagnosed by ophthalmologists from 2011 to 2018 in the eye center of Beijing Tongren Hospital. Follow-up questionnaires were completed for all cases. 
Table 1 Risk factor comparison between congenital microphthalmia and anophthalmia

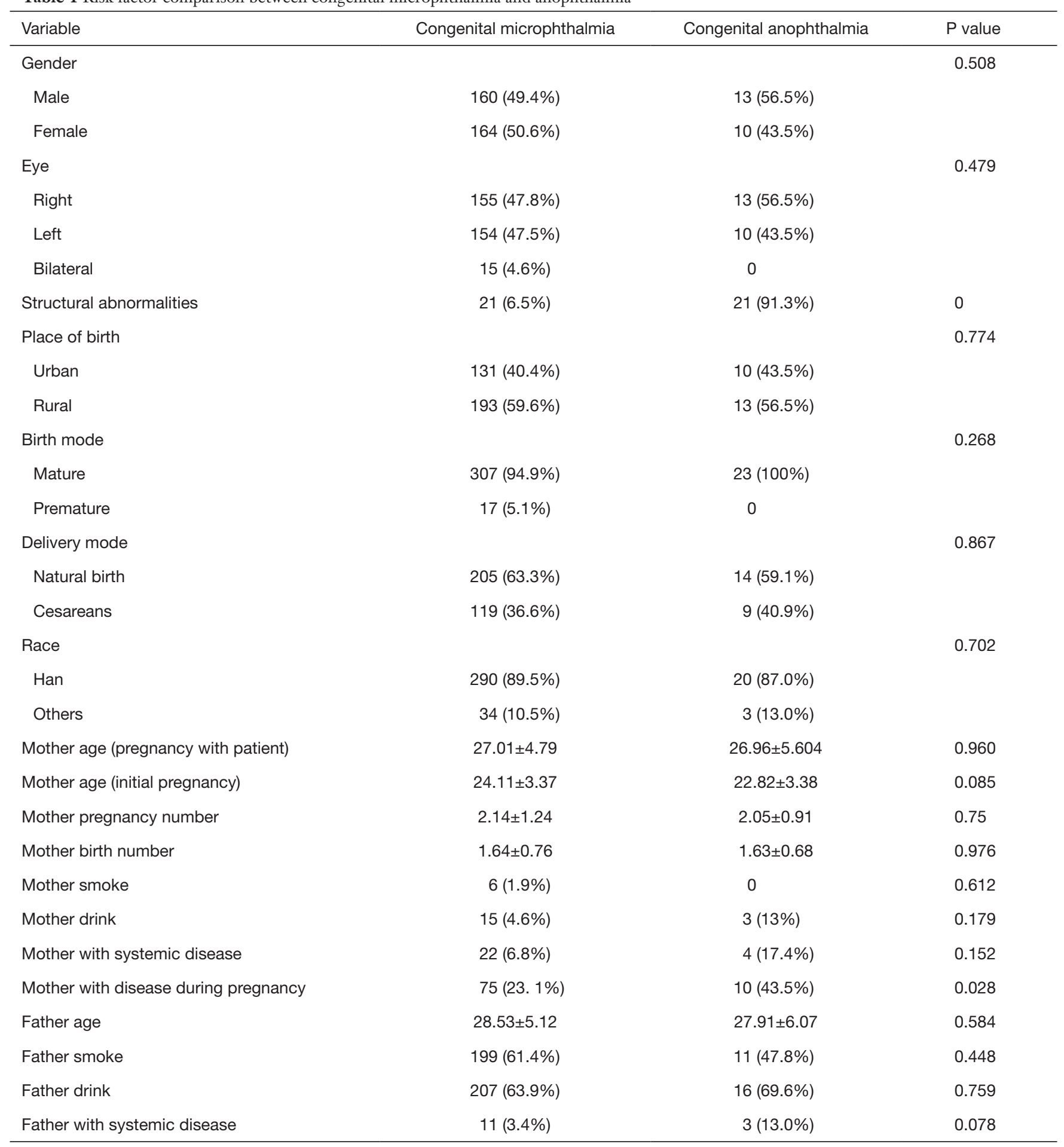

All variables compared between patients with congenital microphthalmia and anophthalmia are included in Table 1. Mother with disease during pregnancy was the only risk factor that was significantly different $(\mathrm{P}=0.028)$. The other factors, including gender, eye, structural abnormalities, place of birth, birth mode, delivery mode, race, mother age 
Table 2 Results of multivariable logistic regression

\begin{tabular}{lccc}
\hline Variable & P value & OR & $95 \% \mathrm{Cl}$ \\
\hline $\begin{array}{l}\text { Mother with disease during } \\
\text { pregnancy }\end{array}$ & 0.023 & 2.804 & $1.151-6.832$ \\
Father with systemic disease & 0.027 & 4.795 & $1.196-19.232$ \\
\hline
\end{tabular}

$\mathrm{OR}$, odds ratios; $\mathrm{Cl}$, confidence intervals.

Table 3 Structural abnormalities in children with congenital microphthalmia

\begin{tabular}{lc}
\hline Structural abnormality & Frequency \\
\hline Polydactyly & 1 \\
Accessory auricle & 1 \\
Otocleisis & 1 \\
Ear deformities & 1 \\
Dysaudia & 1 \\
Bradygenesis & 1 \\
Tetralogy of Fallot & 2 \\
Pulmonary stenosis & 1 \\
Inguinal hernia & 1 \\
Capillary hemangioma of skin & 2 \\
Vertebra abnormal & 1 \\
Syndactyly & 1 \\
Megacolon & 1 \\
Incontinentia pigmenti & 1 \\
Ventricular septal defect & 1 \\
Cleft lip and palate & 1 \\
Congenital hypothyroidism & 1 \\
Cryptorchidism & 1 \\
Total & 1 \\
\hline & 1 \\
\hline
\end{tabular}

(pregnancy with patient), mother age (initial pregnancy), mother pregnancy number, mother birth number, mother smoke, mother drink, mother with systemic disease, mother with disease during pregnancy, father age, father smoke, father drink, father with systemic disease, were not statistically significant. Variables considered important were mother age (initial pregnancy), mother drink, mother with systemic disease, mother with disease during pregnancy, and father with systemic disease and were included in a multivariable logistic regression model to estimate ORs and
95\% CIs.

The OR of congenital anophthalmia to microphthalmia was 2.804 (95\% CI: 1.151-6.832; $\mathrm{P}=0.023$ ) for those patients whose mother had diseases during pregnancy. The OR of congenital anophthalmia to microphthalmia is 4.795 (95\% CI: 1.196-19.232; $\mathrm{P}=0.027$ ) for those patients whose father had systemic diseases (Table 2).

Twenty-one patients with congenital microphthalmia had structural abnormalities (Table 3). These structural abnormalities included polydactyly, accessory auricle, otocleisis, ear deformities, dysaudia, bradygenesis, tetralogy of Fallot, pulmonary stenosis, inguinal hernia, capillary hemangioma of skin, vertebra abnormal, syndactyly, megacolon, incontinentia pigmenti, ventricular septal defect, cleft lip and palate, congenital hypothyroidism and cryptorchidism.

Mother with diseases during pregnancy was an important factor in this study, which was the only factor with statistically significant differences between the two groups. The detailed diseases in each group are listed in Table 4. According to the results, influenza or common cold infection was the most common cause of illness in a mother's pregnancy. Father with systemic diseases was also an important factor. The detailed diseases are listed in Table 5.

\section{Discussion}

In this study, we aimed to analyze the different risk factors between patients with congenital anophthalmia and microphthalmia. This was the first study on risk factors in Chinese patients. By comparing risk factors, we sought to explore the different mechanisms in those two types of cases.

The OR of congenital anophthalmia to microphthalmia was 2.804 (95\% CI: 1.151-6.832; $\mathrm{P}=0.023$ ) for those patients whose mother had diseases during pregnancy. The OR of congenital anophthalmia to microphthalmia was 4.795 (95\% CI: $1.196-19.232 ; \mathrm{P}=0.027$ ) for those patients whose father had systemic diseases. The rate of patients combined with systemic abnormalities is much less than patients who did not have systemic abnormalities.

Many studies have shown that anophthalmia and microphthalmia may have a substantial and overlapping genetic component. Recent studies indicate that approximately $50 \%$ of patients have mutations in more than 20 genes, including but not limited to, SOX2, OTX2, and PAX6 (6). Much less is known about nongenetic risk factors for anophthalmia and microphthalmia $(7,8)$. Previous 
Table 4 Diseases during pregnancy

\begin{tabular}{lcc}
\hline Variable & Frequency & Percentage (\%) \\
\hline Congenital microphthalmia & 1 & 1.33 \\
Fever (unknown cause) & 2 & 2.67 \\
Diarrhea & 1 & 1.33 \\
Dog bite & 1 & 1.33 \\
Appendicitis & 1 & 1.33 \\
Syphilis & 1 & 1.33 \\
Tracheitis & 1 & 1.33 \\
Pregnancy hypertension & 1 & 1.33 \\
Gestational diabetes & 64 & 85.33 \\
Influenza or common cold & 1 & 1.33 \\
Renal calculus & 1 & 1.33 \\
Colpitis & 10 & 100.00 \\
Total & 15 & 10.00 \\
Congenital anophthalmia & & 10.00 \\
Epilepsy & 1 & 100.00 \\
Rubella & 1 & \\
Diarrhea & 1 & \\
Influenza or common cold & 1 & \\
Total & 1 & \\
\hline & 1 & \\
\hline
\end{tabular}

publications have indicated that, similar to other birth defects, anophthalmia and microphthalmia are associated with various risk factors, including elevated maternal prepregnancy body mass index (BMI), maternal smoking during pregnancy, and maternal exposure to medications, including anti-inflammatory drugs and antibiotics before or during early pregnancy $(3,9,10)$. Furthermore, advanced maternal age and maternal history of various viral infections, including rubella and influenza, may increase the likelihood of having a child born with these conditions (11-13). In our study, we compared different risk factors between patients with congenital microphthalmia and anophthalmia. We found that mothers with disease during pregnancy and flu were the most common causes, which was almost consistent with previous studies. Although there are differences in risk factors between our study and previous studies, it is worth noting that we did not compare the patients with the general population, which is different from previous studies. According to this result, we suspected that congenital
Table 5 Systemic diseases of father

\begin{tabular}{lcc}
\hline Variable & Frequency & Percentage (\%) \\
\hline Congenital microphthalmia & 1 & 9.10 \\
Diabetes mellitus type I & 2 & 18.18 \\
Hypertension & 1 & 9.10 \\
Allergies & 1 & 9.10 \\
Chronic pharyngitis & 1 & 9.10 \\
Chronic bronchitis & 1 & 9.10 \\
Renal calculus & 1 & 9.10 \\
Genital herpes & 1 & 9.10 \\
Uarthritis & 1 & 9.10 \\
Gastric cancer & 1 & 9.10 \\
Hepatitis B & 11 & 100.00 \\
Total & & 33.33 \\
Congenital anophthalmia & 1 & 100.00 \\
Hypertension, diabetes & 3 & 33.33 \\
Hepatitis B & & \\
Gastric erosion & 1 & \\
Total & & \\
\hline
\end{tabular}

anophthalmia might be influenced more by nongenetic risk factors during pregnancy than by microphthalmia. However, this is just an assumption; more studies and evidence are needed.

The rate of patients combined with systemic abnormalities is much lower than in previous studies. In our study, systemic abnormalities were recorded in only 13 cases $(7 \%)$ in patients with congenital microphthalmia. None of the cases had systemic abnormalities in anophthalmia. Data on systemic abnormalities in children with congenital microphthalmia and anophthalmia are difficult to compare because studies use different definitions and classification systems, and some studies include stillbirths, neonatal deaths, and terminations of pregnancy, which are likely to have higher rates of major anomalies than studies that only involve live born or living children. In previous studies, data from anomaly registers indicate that the proportion of children with anophthalmia or microphthalmia who also have systemic abnormalities ranges from $52.7 \%$ to $95 \%(14-16)$. The reasons for this difference are not clear but may be methodological in nature. However, several studies have reported that less than $10 \%$ of children with 
anophthalmia and microphthalmia have isolated defects (i.e., born with no additional structural birth defects) $(12,17)$. According to those studies, a substantial majority of infants born with this condition are likely to have other structural birth defects, such as congenital heart disease or oral clefts $(3,17)$. Moreover, previous studies have suggested that over $20 \%$ of children with anophthalmia and microphthalmia have syndromic features, with one or more chromosome abnormalities, such as trisomy 13 or trisomy $18(3,17)$. Our study identified 21 cases with extraocular structural abnormalities in patients with congenital microphthalmia. A limitation in our study, as in most others, was that the children were not all examined in detail by a pediatrician, and thus some systemic findings, particularly milder abnormalities, may have been missed.

The findings described here need to be interpreted in light of several strengths and limitations. First, image studies were taken to make diagnoses for patients with congenital microphthalmia and anophthalmia, which was particularly important for anophthalmia diagnosis. Because there were many "clinical anophthalmia", the patient was appeared to lack an eyeball in the orbit. However, there was actually a very small eyeball in the orbit, which was covered by conjunctiva. The small eyeball could be identified by MRI, CT or ultrasound, especially on MRI or CT scan. Furthermore, we compared risk factors between patients with congenital microphthalmia and anophthalmia; this kind of study was uncommon. However, this study also had limitations. The number of patients with anophthalmia was too few, which might impact the statistical analysis.

In conclusion, this study provides the risk factor for congenital microphthalmia and anophthalmia in Chinese patients for the first time. We found that mothers with disease (mainly influenza or common cold infection) during pregnancy and fathers with systemic disease are risk factors specific for congenital anophthalmia but not microphthalmia. Flu is the most common cause of illness in a mother's pregnancy. The rate of patients combined with systemic abnormalities is much less than patients without systemic abnormalities. Our findings add to the limited body of literature on anophthalmia and microphthalmia.

\section{Acknowledgments}

Funding: This work was supported by the Capital's Funds for Health Improvement and Research, Grant Number: 2018-2-2053 and the Special Fund of the Pediatric Medical Coordinated Development Center of Beijing Hospitals
Authority, Grant Number: XTCX201824.

\section{Footnote}

Provenance and Peer Review: This article was commissioned by the Guest Editors (Haotian Lin and Limin Yu) for the series "Medical Artificial Intelligent Research" published in Annals of Translational Medicine. The article was sent for external peer review organized by the Guest Editors and the editorial office.

Conflicts of Interest: All authors have completed the ICMJE uniform disclosure form (available at http://dx.doi. org/10.21037/atm.2019.12.103). The series "Medical Artificial Intelligent Research" was commissioned by the editorial office without any funding or sponsorship. The authors have no other conflicts of interest to declare.

Etbical Statement: The authors are accountable for all aspects of the work in ensuring that questions related to the accuracy or integrity of any part of the work are appropriately investigated and resolved. This study was approved by the Ethics Committee of Beijing Tongren Hospital and adhered to the provisions of the Declaration of Helsinki for research involving human subjects. Written informed consent was obtained from the patient for publication of this study and any accompanying images. A copy of the written consent is available for review by the Editor-in-Chief of this journal.

Open Access Statement: This is an Open Access article distributed in accordance with the Creative Commons Attribution-NonCommercial-NoDerivs 4.0 International License (CC BY-NC-ND 4.0), which permits the noncommercial replication and distribution of the article with the strict proviso that no changes or edits are made and the original work is properly cited (including links to both the formal publication through the relevant DOI and the license). See: https://creativecommons.org/licenses/by-nc-nd/4.0/.

\section{References}

1. Verma AS, Fitzpatrick DR. Anophthalmia and microphthalmia. Orphanet J Rare Dis 2007;2:47.

2. Traboulsi EI. Developmental genes and ocular malformation syndromes. Am J Ophthalmol 1993;115:105-7.

3. Källén B, Tornqvist K. The epidemiology of anophthalmia 
and microphthalmia in Sweden. Eur J Epidemiol 2005;20:345-50.

4. Boyd PA, Armstrong B, Dolk H, et al. Congenital anomaly surveillance in England--ascertainment deficiencies in the national system. BMJ 2005;330:27.

5. Morrison D, FitzPatrick D, Hanson I, et al. National study of microphthalmia, anophthalmia, and coloboma (MAC) in Scotland: investigation of genetic aetiology. J Med Genet 2002;39:16-22.

6. You T, Lv Y, Liu S, et al. Novel OTX2 mutation associated with congenital anophthalmia and microphthalmia in a Han Chinese family. Acta Ophthalmol 2012;90:e501-2.

7. Plaisancie J, Calvas P, Chassaing N. Genetic Advances in Microphthalmia. J Pediatr Genet 2016;5:184-8.

8. Williamson KA, FitzPatrick DR. The genetic architecture of microphthalmia, anophthalmia and coloboma. Eur J Med Genet 2014;57:369-80.

9. Crider KS, Cleves MA, Reefhuis J, et al. Antibacterial medication use during pregnancy and risk of birth defects: National Birth Defects Prevention Study. Archives of Pediatrics \& Adolescent Medicine 2009;163:978-85.

10. Shaw GM, Carmichael SL, Yang W, et al. Epidemiologic characteristics of anophthalmia and bilateral microphthalmia among 2.5 million births in California, 1989-1997. Am J Med Genet A 2005;137:36-40.

Cite this article as: Li Y, Hou Z, Ding J, Cui Y, Qin B, Li D. Distinguishing risk factors between congenital anophthalmia and microphthalmia using multivariable logistic regression. Ann Transl Med 2020;8(11):704. doi: 10.21037/atm.2019.12.103
11. Busby A, Dolk H, Armstrong B. Eye anomalies: Seasonal variation and maternal viral infections. Epidemiology 2005;16:317-22.

12. Forrester MB, Merz RD. Descriptive epidemiology of anophthalmia and microphthalmia, Hawaii, 1986-2001. Birth Defects Research Part A: Clinical and Molecular Teratology 2006;76:187-92.

13. Vogt G, Puho E, Czeizel AE. A population-based case-control study of isolated anophthalmia and microphthalmia. Eur J Epidemiol 2005;20:939-46.

14. Bermejo E, Martinez-Frias ML. Congenital eye malformations: clinical-epidemiological analysis of $1,124,654$ consecutive births in Spain. Am J Med Genet 1998;75:497-504.

15. Clementi M, Tenconi R, Bianchi F, et al. Congenital eye malformations: a descriptive epidemiologic study in about one million newborns in Italy. Birth Defects Orig Artic Ser 1996;30:413-24.

16. Källén B, Robert E, Harris J. The descriptive epidemiology of anophthalmia and microphthalmia. Int J Epidemiol 1996;25:1009-16.

17. Stoll C, Dott B, Alembik Y, et al. Associated malformations among infants with anophthalmia and microphthalmia. Birth Defects Research Part A: Clinical and Molecular Teratology 2012;94:147-52. 